BMC

Genomics

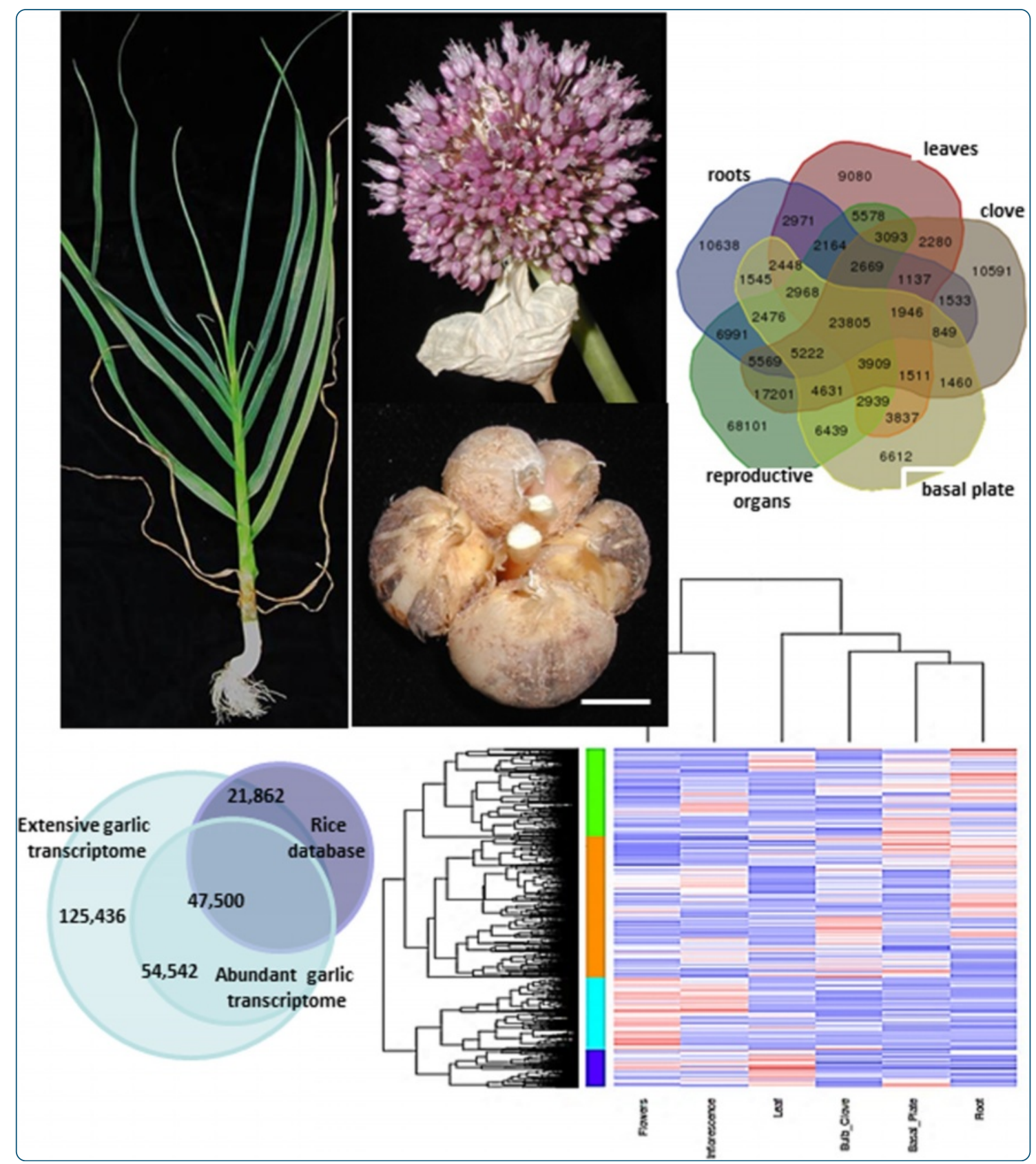

\title{
Integrated transcriptome catalogue and organ-specific profiling of gene expression in fertile garlic (Allium sativum L.)
}

Kamenetsky et al. 


\title{
Integrated transcriptome catalogue and organ-specific profiling of gene expression in fertile garlic (Allium sativum L.)
}

Rina Kamenetsky ${ }^{1 *}$, Adi Faigenboim ${ }^{1}$, Einat Shemesh Mayer ${ }^{1,2}$, Tomer Ben Michael $^{1,2}$, Chen Gershberg ${ }^{1}$, Sagie Kimhi ${ }^{1}$, Itzhak Esquira ${ }^{3}$, Sarit Rohkin Shalom ${ }^{1,4}$, Dani Eshel ${ }^{4}$, Haim D Rabinowitch ${ }^{2}$ and Amir Sherman ${ }^{1}$

\begin{abstract}
Background: Garlic is cultivated and consumed worldwide as a popular condiment and green vegetable with medicinal and neutraceutical properties. Garlic cultivars do not produce seeds, and therefore, this plant has not been the subject of either classical breeding or genetic studies. However, recent achievements in fertility restoration in a number of genotypes have led to flowering and seed production, thus enabling genetic studies and breeding in garlic.

Results: A transcriptome catalogue of fertile garlic was produced from multiplexed gene libraries, using RNA collected from various plant organs, including inflorescences and flowers. Over 32 million 250-bp paired-end reads were assembled into an extensive transcriptome of 240,000 contigs. An abundant transcriptome assembled separately from 102,000 highly expressed contigs was annotated and analyzed for gene ontology and metabolic pathways. Organ-specific analysis showed significant variation of gene expression between plant organs, with the highest number of specific reads in inflorescences and flowers. Analysis of the enriched biological processes and molecular functions revealed characteristic patterns for stress response, flower development and photosynthetic activity. Orthologues of key flowering genes were differentially expressed, not only in reproductive tissues, but also in leaves and bulbs, suggesting their role in flower-signal transduction and the bulbing process. More than 100 variants and isoforms of enzymes involved in organosulfur metabolism were differentially expressed and had organ-specific patterns. In addition to plant genes, viral RNA of at least four garlic viruses was detected, mostly in the roots and cloves, whereas only $1-4 \%$ of the reads were found in the foliage leaves.
\end{abstract}

Conclusions: The de novo transcriptome of fertile garlic represents a new resource for research and breeding of this important crop, as well as for the development of effective molecular markers for useful traits, including fertility and seed production, resistance to pests and neutraceutical characteristics.

Keywords: Assembly, Flowering, Breeding, Sulfur metabolism, Garlic virus

\section{Background}

Garlic (Allium sativum L.), the second most important Allium crop after the bulb onion, is cultivated worldwide and consumed by almost every culture as a popular condiment and green vegetable. It is also known for its medicinal and neutraceutical properties, with a large spectrum of antibacterial and anti-inflammatory activity [1-3]. None of the known commercial garlic cultivars and landraces

\footnotetext{
* Correspondence: vhrkamen@volcani.agri.gov.il

'Institute of Plant Sciences, ARO, The Volcani Center, Bet Dagan, Israel

Full list of author information is available at the end of the article
}

produce fertile flowers or seeds. Therefore, garlic is not propagated sexually, and neither classical breeding nor genetic studies are currently employed with this plant. Nevertheless, over the past 20 years, fertility restoration has been achieved in a number of garlic genotypes, mostly landraces from Central Asia [4-8], and physiological studies have led to flowering induction and seed production by environmental manipulation [8,9]. Self- and cross-pollination within and between garlic genotypes has become a reality, thus enabling the initiation of genetic studies and classical breeding in garlic [2,10-14].

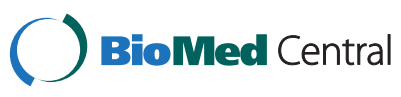

(c) 2015 Kamenetsky et al.; licensee Biomed Central. This is an Open Access article distributed under the terms of the Creative Commons Attribution License (http://creativecommons.org/licenses/by/4.0), which permits unrestricted use, distribution, and reproduction in any medium, provided the original work is properly credited. The Creative Commons Public Domain Dedication waiver (http://creativecommons.org/publicdomain/zero/1.0/) applies to the data made available in this article, unless otherwise stated. 
Garlic generates the highest known concentration of organosulfur compounds among Allium species. These are responsible for both garlic's pungent odor and many of its medicinal benefits. The levels of the flavor precursors, non-protein cysteine and glutathione derivatives, account for $1-5 \%$ of the garlic's dry weight [15], indicating the major importance of organosulfur biosynthetic activity within the plants' tissues. These compounds serve two roles in the life cycle of the plant: pest deterrence and the storage and transport of carbon, nitrogen, and sulfur [15]. The major garlic flavor precursor is sulfur-alk(en)yl cysteine sulfoxide (alliin). This chemical is broken down by alliinase to pyruvate, ammonia and thiosulfinate allicin, which are the sources of the typical aroma and proposed health-beneficial properties of garlic $[3,16,17]$.

Like many perennial monocots, edible Allium species have very large genome sizes of 10-20 Gbp [18]. The diploid garlic $(2 \mathrm{n}=2 \mathrm{x}=16)$ nuclear genome is estimated at $15.9 \mathrm{Gbp}, 32$ times larger than the genome of rice [19]. Therefore, full sequencing of the garlic genome is a challenging task, but transcriptome assembly using next generation sequencing might be efficiently employed for the generation of functional genomic data. This approach has been successfully applied to perennial crops, e.g., peony [20], pomegranate [21], orchid [22] and Arundo donax [23], as well as to the bulbous monocots Tulipa and Lilium [24,25] and onion [26]. In garlic, the first transcriptome analysis based on RNA from the renewal buds resulted in de novo assembly of 128,000 unigenes which were annotated and analyzed with respect to gene ontology (GO) and metabolic pathways [27]. In addition, 352 differentially expressed transcript-derived fragments showed differential expression in the leaf, meristematic and flower tissues [28].

Since only a few garlic genotypes are able to produce flowers and viable seeds, getting this plant to flower is still a challenge, and genetic regulation of the reproductive process is of special interest [2]. Recent physiological studies have facilitated flowering induction and fertility restoration by environmental manipulations in a variety of garlic genotypes [9,11,13], and our research group currently maintains numerous genotypes of fertile seedproducing garlic genotypes. A further and deeper understanding of the genetic make-up of garlic reproductive traits is required for both improved genetic and physiological knowledge and exploitation of the genetic potential of this important crop.

In this report, we describe the de novo assembly of a transcriptome catalogue for fertile garlic, and provide a few examples of its analysis. This is the first reported garlic transcriptome representing genes expressed in vegetative and reproductive organs, which were sampled and analyzed throughout plant development, from clove sprouting to flowering and bulbing. The results provide a platform for further research and breeding of this important crop.

\section{Results and discussion}

\section{Transcriptome sequencing and annotation}

To establish a transcriptome catalogue for fertile garlic, we used MiSeq pair-end technology, which enabled us to create an assembly of longer contigs. While the longread protocol is considerably more expensive than shortread sequencing, important benefits include a lower mapping bias and reduced ambiguity in assigning reads to genomic elements, such as mRNA transcripts [29]. Six cDNA libraries from six garlic organs yielded approximately 32.6 million long 250 -bp paired-end reads (Table 1). Quality trimming and filtration resulted in 28.4 million cleaned reads that were assembled using Trinity, and generated 239,116 contigs for the "extensive transcriptome catalogue". The average contig length was $715 \mathrm{bp}$; half of these (N50) were at least 1,088 bp long. The elimination of contigs with fewer than 10 total mapped reads resulted in a further reduction, resulting in a total of 102,042 contigs, assembled separately into an "abundant transcriptome catalogue". Clustering the sequences with 95\% identity revealed a redundancy of less than $10 \%$ in both the extensive and abundant transcriptomes.

Both transcriptome catalogues of garlic were compared with the database of Oryza sativa-the most sequenced and annotated monocot species (Figure 1). BLASTX search against the rice database resulted in at least one significant hit for 59,138 contigs of the extensive garlic transcriptome (24.7\%); 47,500 of those contigs represented $46.5 \%$ of the contigs of the abundant transcriptome. In a reciprocal TBLASTN search of the rice genes in the extensive garlic transcriptome, at least one significant hit was found for 44,476 rice sequences, i.e., $67 \%$ of the rice sequences were found in garlic (Figure 1).

Comparison of the abundant garlic transcriptome with public databases revealed various levels of similarity with the model species Arabidopsis and Oryza, as well as other bulbous geophytes (Table 2). Databases of Arabidopsis, rice and nrNCBI showed a general similarity of $45-47 \%$ with our data. In addition, the nrNCBI protein database was used to search for the five top hits. Among the 47,509 contigs annotated in the abundant transcriptome, $27 \%$ show high similarity to Oryza sativa, $8.4 \%$ to Hordeum, 5.52\% to Triticum, and 8\% to Arabidopsis (search results from April 2014). The main reason for such similarity might be higher representation of the genomic resources for these species. There were abundant transcriptome matches with 463 published genes from 10 Allium species and 458 genes from 33 geophyte species that were closely or remotely related to garlic 
Table 1 Statistical summary of extensive and abundant transcriptome catalogues of the fertile garlic

\begin{tabular}{llllllll}
\hline & Total in plant & Root & Basal plate & Leaf & Clove & Inflorescence & Flowers \\
\hline Read pairs & $32,607,803$ & $5,460,636$ & $5,654,667$ & $4,228,289$ & $5,539,119$ & $6,969,526$ & $4,755,566$ \\
Cleaned read pairs & $28,430,270$ & $4,737,985$ & $5,007,820$ & $3,729,942$ & $4,777,731$ & $6,013,904$ & $4,162,888$ \\
Extensive transcriptome & 239,116 & 104,937 & 111,289 & 85,423 & 144,405 & 236,946 & 203,781 \\
Unique components & 183,328 & 75,592 & 74,221 & 62,676 & 92,782 & 129,965 & 122,490 \\
Abundant Transcriptome* & 102,042 & 30,390 & 33,155 & 25,109 & 39,119 & 62,678 & 53,066 \\
\hline
\end{tabular}

cDNA libraries were sampled from six vegetative and reproductive organs.

${ }^{*}$ Contigs computed with count per sample $>10$.

(e.g., Lilium, Narcissus, Tulipa, and Agapanthus). Of the contigs obtained here, 44 corresponded with at least 4 known garlic viruses.

Although $78 \%$ of the sequences were homologous with the previously reported transcriptome of garlic renewal bud [27], reads of our transcriptome were much longer (N50 of 1,088 bp) than those of this previous report (based only on 90 bp paired-end reads), and therefore represented larger portions of the full genes. In addition, BLASTN analysis identified $51-58 \%$ similarity of the reference transcriptome with onion accessions [26]. It is interesting to note that the similarity between the abundant catalogue and the published sequences of bulbous monocots Lilium and Tulipa [24,25] was rather lowonly 21.3 and $20.6 \%$, respectively. However, taking into account that reports for Lilium and Tulipa include only the leaf transcriptome, we repeated the BLASTN analysis with the 25,109 contigs specific to garlic leaves. In this case, much higher match was obtained, 45.3 and $44.5 \%$ for Lilium and Tulipa, respectively. In comparison, the resemblance between rice and the annotated transcriptomes of Lilium and Tulipa reaches $49 \%$ and $30 \%$, respectively [24]. This clearly demonstrates that comparative analyses between transcriptomes that include samples from various organs at different stages of plant development provide more reliable results. Transcriptome analysis from only one plant organ or tissue does not provide a full transcript catalogue, even though it can serve numerous specific genetic and breeding objectives [25].

Comparative phylogeny of monocots has suggested that the divergence between Liliales (e.g., Tulipa and Lilium) and Asparagales (Allium) occurred between 120 and 133 million years ago, while divergence between Poales (Oryza) and Asparagales took place later, between 98 and 116 million years ago [30,31]. Although Asparagales and Liliales have evolved many traits in parallel and it is difficult to separate them morphologically, gene-sequencing analysis supports these two orders as two separate monophyletic groups [32]. Rice might be phylogenetically closer to Asparagales than to Liliales, and therefore, only a relatively low number of shared

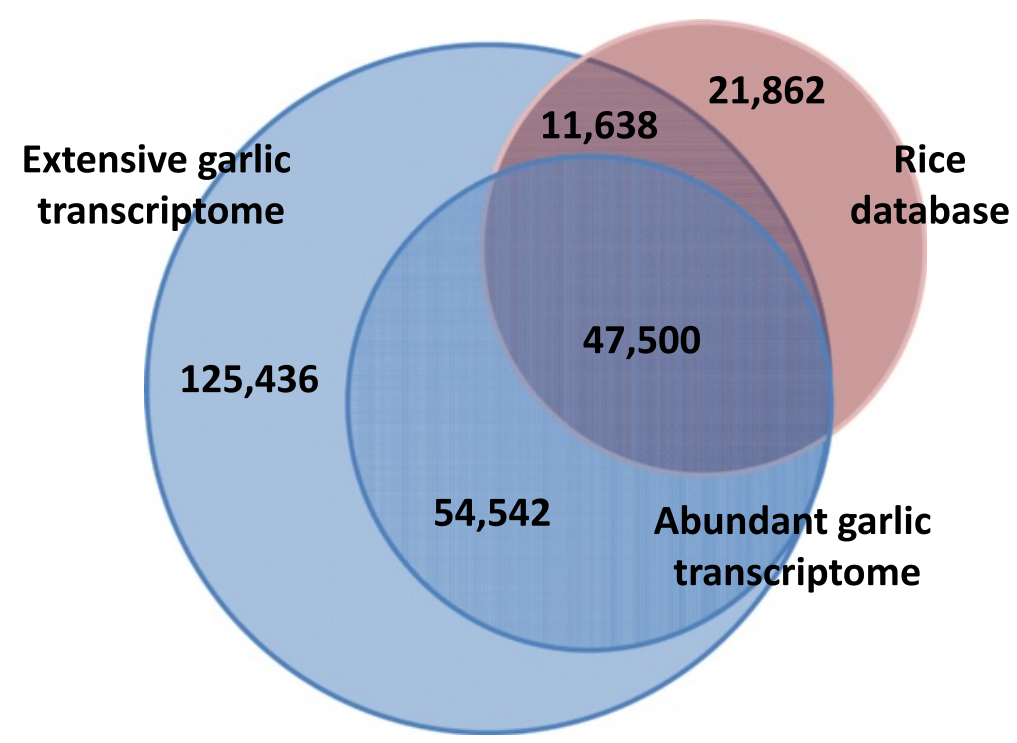

Figure 1 Venn diagram of the distribution and similarity of sequences in extensive and abundant transcriptome catalogues of garlic in comparison with rice protein database (www.phytozome.org). 
Table 2 Global sequence similarity of garlic abundant transcriptome to transcriptome databases for model and related species

\begin{tabular}{llll}
\hline Reference & Reference database & $\begin{array}{l}\text { Number of reference } \\
\text { sequences }\end{array}$ & $\begin{array}{l}\text { Percent of hits in } \\
\text { reference database }\end{array}$ \\
\hline nrNCBI & http://www.ncbi.nlm.nih.gov & $\sim 169.000 .000$ & 46.6 \\
Arabidopsis & http://www.arabidopsis.org & 41,671 & 45.8 \\
Oryza sativa & www.phytozome.org & 66,338 & 46.6 \\
Allium cepa & http://www.ncbi.nlm.nih.gov PRJNA175446 & 26,995 & 57.8 \\
Allium cepa & http://www.ncbi.nlm.nih.gov PRJNA175449 & 33,162 & 51.3 \\
Allium sativum (renewal bud) & http://www.ncbi.nlm.nih.gov PRJNA158177 & 79,143 & 77.8 \\
Tulipa (leaves) & {$[24,25]$} & 52172 & $44.5^{*}$ \\
Lilium (leaves) & {$[24,25]$} & 79980 & $45.3^{*}$ \\
\hline
\end{tabular}

*Based on 25,109 sequences specific to garlic leaves.

possible orthologous groups are identified between rice, garlic, Tulipa and Lilium. On the other hand, the low ratio of similarity demonstrates a potential source of unidentified specific genes that need to be annotated in bulbous monocots. It is also possible that genes from bulbous monocots deviate at the sequence level from the allied genes existing in the databases, or that some of the transcripts in the published transcriptomes were not long enough to find a significant similarity with annotated genes.

\section{Organ-specific expression analysis}

Analysis of the extensive transcriptome showed that most contigs are shared between plant organs, but only $13.7 \%$ $(23,805)$ are common to all studied organs (Figure 2). On the other hand, numerous contigs were organ-specific, i.e., to the roots $(10,638)$, leaves $(9,080)$, basal plate $(6,612)$ and clove tissue $(10,591)$. Similarly, large differences in gene-expression patterns between different tissues, especially between root and aerial organs, have been reported for other plants, e.g. Arabidopsis [33] and Glycine max [34]. In rice, $69 \%$ of the expressed transcripts show significantly variable expression levels among tissues/organs [35]. In our study, the number of specific reads was exceptionally high in the reproductive organs-68,101 transcripts expressed only in inflorescences and flowers, representing $28.5 \%$ of the extensive transcriptome-thus confirming that the signaling and transcriptional control of reproductive development involves a number of regulatory mechanisms and a large number of genes. Similarly, 25,062 pollen-preferential transcripts are expressed in rice pollen [36], and 1,145 soybean genes are flower-specific [34]. Detailed transcriptome comparisons between different species may reveal evolutionarily conserved genes and molecular mechanisms in plant reproduction processes. Such comparisons require comprehensive transcriptome datasets of tissues and developmental stages, and precise annotations of orthologous genes between species [37].
Global gene-expression profiling of the abundant transcriptome revealed differential patterns between the vegetative and reproductive organs (Figure 3 ). The vegetative organs could be clustered into two defined groups, with the underground organs-roots and basal plate-sharing both close proximity and many expressed sequences. In bulbous geophytes, the basal plate (the compressed actual stem) is morphologically associated with the roots, leaves and renewal buds [38]. When the reproductive stage begins, an apical meristem forms the floral scape and inflorescence. Flower differentiation in garlic is affected by endogenous and environmental signals. However, it is not clear whether florogenesis is regulated by direct signal transduction from the vegetative organs, or is only supported by photosynthetic products and storage materials from foliage leaves and the underground bulb $[9,13]$.

Hierarchical cluster analysis of gene-expression patterns revealed four significantly different groups of contigs (Figure 3). Alterations in gene expression provided information on organography of the expression patterns.

Cluster \#1 (highlighted in green in Figure 3) consisted of 2,439 transcripts, enriched mainly in vegetative organs. Employing GO enrichment analysis in this cluster revealed gene-expression patterns reflecting intense metabolic activity, transport, and regulation of the stress response (Additional file 1: Figure S1a). Biological processes related to responses to external stimuli, such as inorganic and organic substances, and to various types of abiotic stress, such as osmotic, oxidative or salt stress, were prominent in the roots and basal plate. Regulation of cellular response to stress in the roots might be induced by endogenous stimuli, by interactions with symbionts and pathogens, or by wounding. The main metabolic functions of cluster \#1 included antioxidant activities, especially oxidoreductase, reductase, and carbon-sulfur lyase activities. In plants, the deleterious effect of reactive oxygen species (ROS) accounts for many of the stress conditions and abiotic damage. To protect against injury by free 


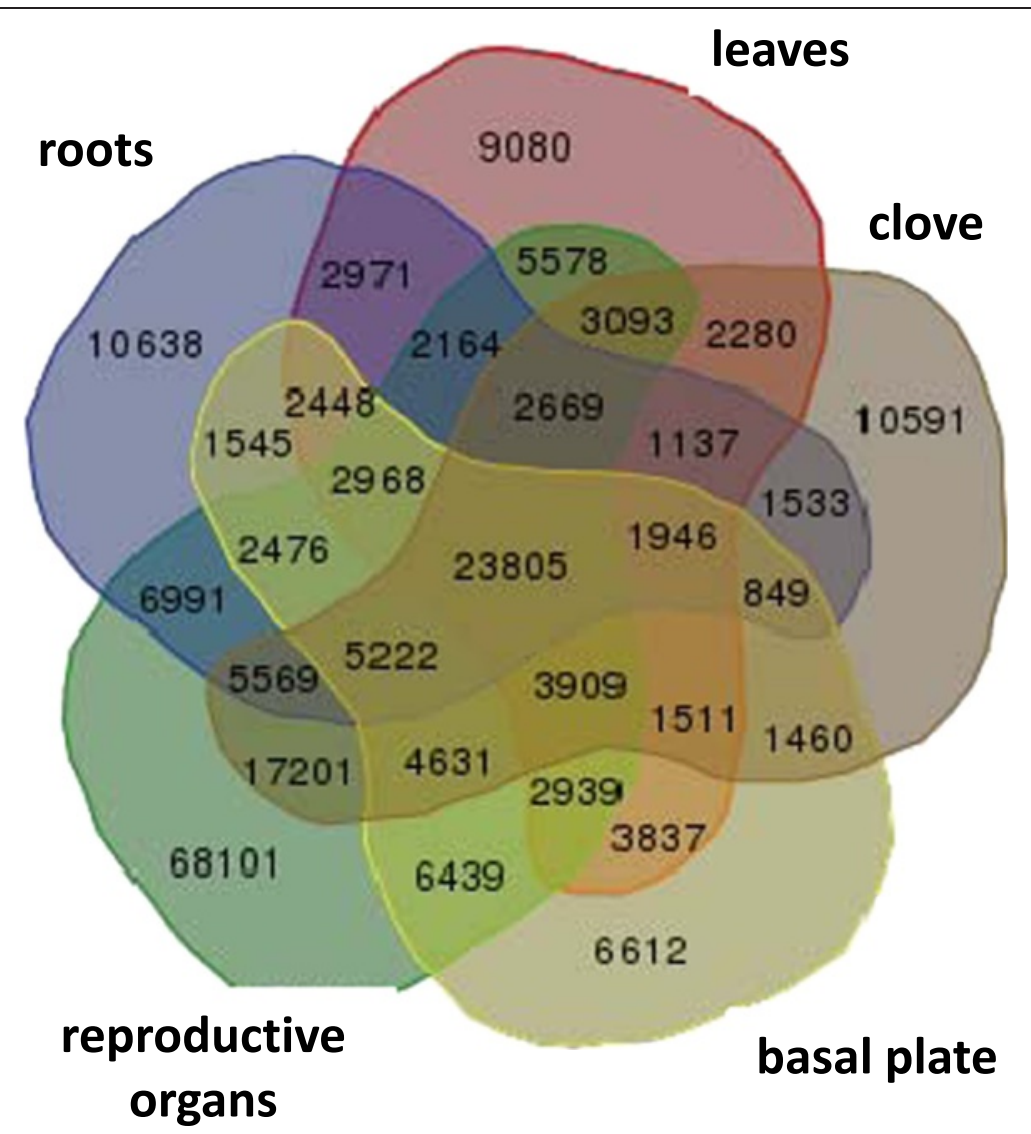

Figure 2 Common and specific contigs found in the extensive transcriptome catalogue of the various organs of fertile garlic. Note the high number of specific contigs in the reproductive tissues. Samples of the reproductive organs (young inflorescences and flowers at various stages of development) are unified for this analysis.

radicals, living organisms have developed a battery of antioxidant defenses, such as the enzymes superoxide dismutase (SOD), catalase and glutathione peroxidase [39]. In addition, small molecules, including glutathione and other organosulfur compounds, play a significant role in the defense against ROS effects. It is therefore suggested that roots might play a substantial role in stress resistance and antioxidant defense in garlic plants.

Cluster \#2 (highlighted in orange in Figure 3) contained 3,878 contigs, with a slightly higher ratio of enriched genes in the cloves and roots. However, only a small number of the genes in this cluster were organspecific. Molecular functions, analyzed by the GO test tool, showed enrichment in GO terms involved in catalytic activity, mainly related to carbohydrate transferases. These findings certainly support the concept of carbohydrate accumulation in the storage organs during growth and dormancy induction in garlic $[2,40]$ and other geophytes [41]. On the other hand, analysis of cluster \#2 clearly showed over-representation of genes involved in plant-pathogen interactions, including processes related to viral genome replication and symbiosis between viruses and the garlic plant (Additional file 1: Figure S1b).

Cluster \#3 (highlighted in blue in Figure 3) contained 2,007 contigs. Numerous genes, mostly specific to the reproductive process, were abundant (Figure 2, Additional file 2: Figure S2a). The major processes encoded by these genes in the reproductive tissues involved floral morphogenesis, development of male and female reproductive organs, cell division, and gametophyte development. At least $100 \mathrm{GO}$ terms were significantly enriched in the reproductive organs. Nevertheless, GO analyses showed a variety of biological processes, including DNA replication and modification, regulation of RNA synthesis, cell cycle, cellular component organization activity and more (Additional file 2: Figure S2a). At the cellular level, these processes were associated with cytoskeleton and microtubule activities and chromosomes (data not shown). GO terms of flower morphogenesis, pollen formation, cell cycle and organelle organization were notable (Additional file 2: Figure S2a). This variety might be justified by the wide range of developmental stages sampled: reproductive organ samples were collected from very 


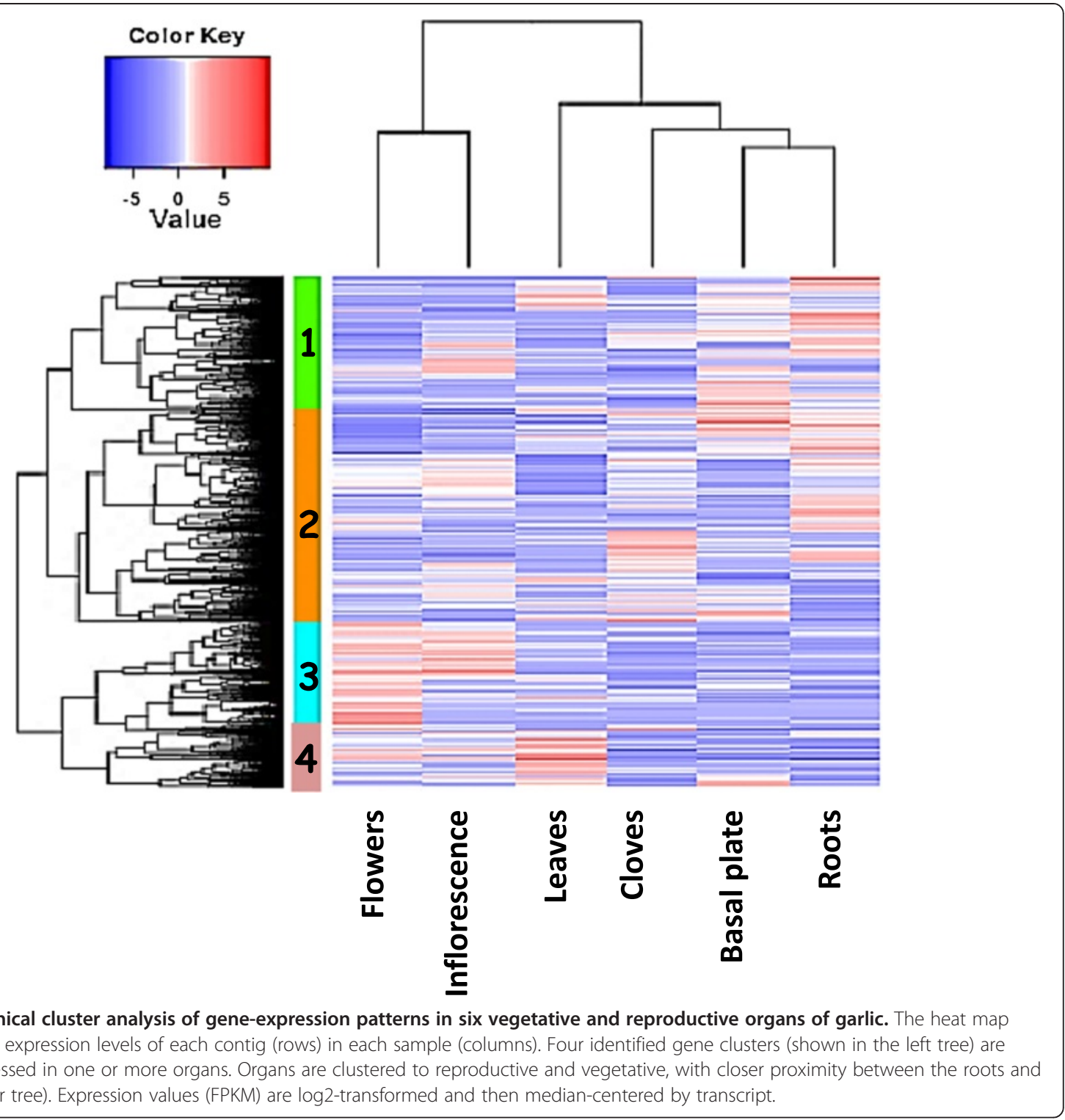

young inflorescences with undifferentiated flower primordia, through flower buds with developing anthers and gynoecia, to fully developed flowers at anthesis.

Cluster \#4 (highlighted in pink in Figure 3) was relatively small, consisting of 1,011 contigs with significant representation in the foliage leaves. Carbohydrate metabolic activities and photosynthetic processes were notable (Additional file 2: Figure S2b). In addition, the metabolism and transport of sulfur, phosphorus, and nitrogen were prominent. Genes associated with synthesis of lipid, sugar and aromatic compounds were over-represented, and expression related to metabolic activity was high. In terms of cellular localization, the processes occurred in most organelles, as well as in membranes and intracellular spaces.

Of the 12 most abundant proteins in the abundant transcript catalogue, 5 matched known sequences from onion and garlic, and another 4 were highly similar to proteins reported from Medicago, Vitis and Nelumbo. Three proteins with highest expression in the leaves and flowers were unidentified (Additional file 3: Table S1).

To date, the identification of genes controlling garlic development or metabolic pathways has been based on a restricted number of gene orthologues [11,14,28]. Establishment of the garlic transcriptome allows for large-scale identification of genes showing tissue-specific expression.

\section{Orthologues of key flowering genes and their expression in garlic organs}

Orthologues of several key flowering genes (Table 3) were found in the transcriptome by matching with known gene sequences from Arabidopsis, Oryza and Allium 
Table 3 Orthologues of key flowering genes and their relative expression in garlic vegetative and reproductive organs

\begin{tabular}{|c|c|c|c|c|c|c|c|}
\hline \multirow[t]{2}{*}{ Flowering genes } & \multirow{2}{*}{$\begin{array}{l}\text { Comp } \\
\text { number }\end{array}$} & \multicolumn{6}{|c|}{ Relative expression in different organs } \\
\hline & & Roots & Basal plate & Cloves & Leaves & Inflorescence & Flowers \\
\hline FLOWERING LOCUS T (FT) & comp149377 & 0 & 0 & 0 & 0 & 1.11 & 3.21 \\
\hline CONSTANS $(C O)^{*}$ & comp45555 & 0.72 & 5.99 & 12.59 & 5.49 & 6.87 & 5.12 \\
\hline SUPPRESSOR OF OVEREXPRESSION OF CONSTANS1 (SOC1)* & comp151688 & 0 & 0 & 0 & 1.26 & 1.13 & 0 \\
\hline LEAFY (LFY) & comp44757 & 1.61 & 0 & 0.77 & 0 & 25.35 & 1.65 \\
\hline APETALA1 (AP1) & comp38587 & 2.52 & 62.14 & 0 & 50.43 & 64.89 & 5.56 \\
\hline APETALA2 (AP2)* & comp46193 & 3.15 & 1.45 & 8.64 & 0.98 & 0.54 & 22.46 \\
\hline APETALA3 (AP3) & comp35626 & 0 & 0 & 0.57 & 0 & 40.93 & 245.92 \\
\hline PISTILLATA (PI) & comp44196 & 0.56 & 0.55 & 0.27 & 0 & 2.58 & 143.66 \\
\hline SEPALLATA1 (SEP1)* & comp56369 & 0 & 0.93 & 0 & 0 & 86.97 & 13.48 \\
\hline SEPALLATA3 (SEP3) & comp42485 & 0.52 & 0 & 0 & 0 & 26.16 & 263.92 \\
\hline AGAMOUS (AG)* & comp59363 & 0.9 & 0 & 0 & 0 & 4.83 & 185.44 \\
\hline
\end{tabular}

Sequences of known flowering genes from public databases were matched with the garlic abundant transcriptome. The expression level (FPKM) was calculated using the expectation maximization method, and TMM normalization (trimmed mean of M-value normalization method) was applied (see Materials and Methods). *Numerous sequences with significant similarity were identified.

cepa databases (Additional file 4: Table S2). In model plants, the floral-induction pathways have been shown to be integrated into a flowering network, which contains several steps [42,43]. Thus, in Arabidopsis, these pathways involve an array of transcription factors [e.g., CONSTANS (CO), FLOWERING LOCUS C (FLC), SUPPRESSOR OF OVEREXPRESSION OF CONSTANS1 (SOC1), LEAFY (LFY), APETALA1 (AP1)], regulators of chromatin structure [VERNALISATION2 (VRN2)], the putative kinase inhibitor $F T$, and many other genes $[44,45]$. The key genes controlling flowering in Arabidopsis are conserved in other species. For instance, in onion, some of these genes are involved not only in the flowering process, but also in the photoperiodic control of bulb initiation $[46,47]$. Our analysis of gene expression in various organs of garlic demonstrated that orthologues of flowering genes can be involved in numerous biological processes, including flower initiation, stem elongation and bulbing.

Numerous isoforms identified for some of the key flowering genes, e.g., FT, $A P 2, S E P 1$ and $A G$, varied in their expression in garlic organs. As an example, at least four sequences of FT orthologues were differentially expressed, with significant over-representation in inflorescences and flowers (Figure $4 \mathrm{a}, \mathrm{b}, \mathrm{c}$ ), roots and basal plate (Figure $4 \mathrm{~b}, \mathrm{~d}$ ), or cloves (Figure $4 \mathrm{c}$ ). FT homologues are involved in a range of plant developmental processes. In perennial tomato, the orthologue SFT has been shown to regulate diverse growth processes, such as the flowering, growth and termination cycles typical of perennial plants, leaf maturation, growth of stems, and the formation of abscission zones [48]. FT homologues are involved in leaf development and flower induction in Narcissus [49] and tuberization in potato
[50]. In onion, at least four different $F T$ genes regulate flowering and bulb formation. Flowering is promoted by vernalization and correlates with upregulation of AcFT2, whereas bulb formation is regulated by two antagonistic FT-like genes [47]. FT orthologues identified in the garlic transcriptome demonstrate strong homology to the onion FT-like genes and might similarly regulate developmental processes in garlic.

Although garlic genetics is still in its infancy, we believe that new transcriptome data will significantly accelerate research into the genetic regulation of flowering, seed development, dormancy induction and the bulbing process in this important crop. Mining for molecular markers for flowering and fertility is of special interest, since their development will certainly advance our progress toward hybridization and seed propagation in garlic.

\section{GO classification in tissue-specific sulfur metabolism}

Garlic has a high content of organosulfur compounds, but little information is currently available on the molecular regulation of sulfur biosynthesis in this plant [3]. Biosynthetic pathways of alliin precursors involve alk(en)ylation of the amino acid cysteine in glutathione, (thio)alk(en)ylation of cysteine or a precursor such as $\mathrm{O}$-acetylserine. The regulatory complex of cysteine synthase is formed by the enzymes O-acetylserine sulfhydrylase (acetylserine(thiol)-lyase (OASS) and serine acetyltransferase (SAT). These enzymes are at the branch point of the sulfur-, carbon-, and nitrogen-assimilation pathways [51]. In garlic, cysteine and glutathione metabolism occurs in parallel, and variation in metabolic routes depends on the physiological state of the tissue $[16,17]$.

Using de novo assembly of the garlic transcriptome, we distinguished about 100 variants and isoforms of nine 

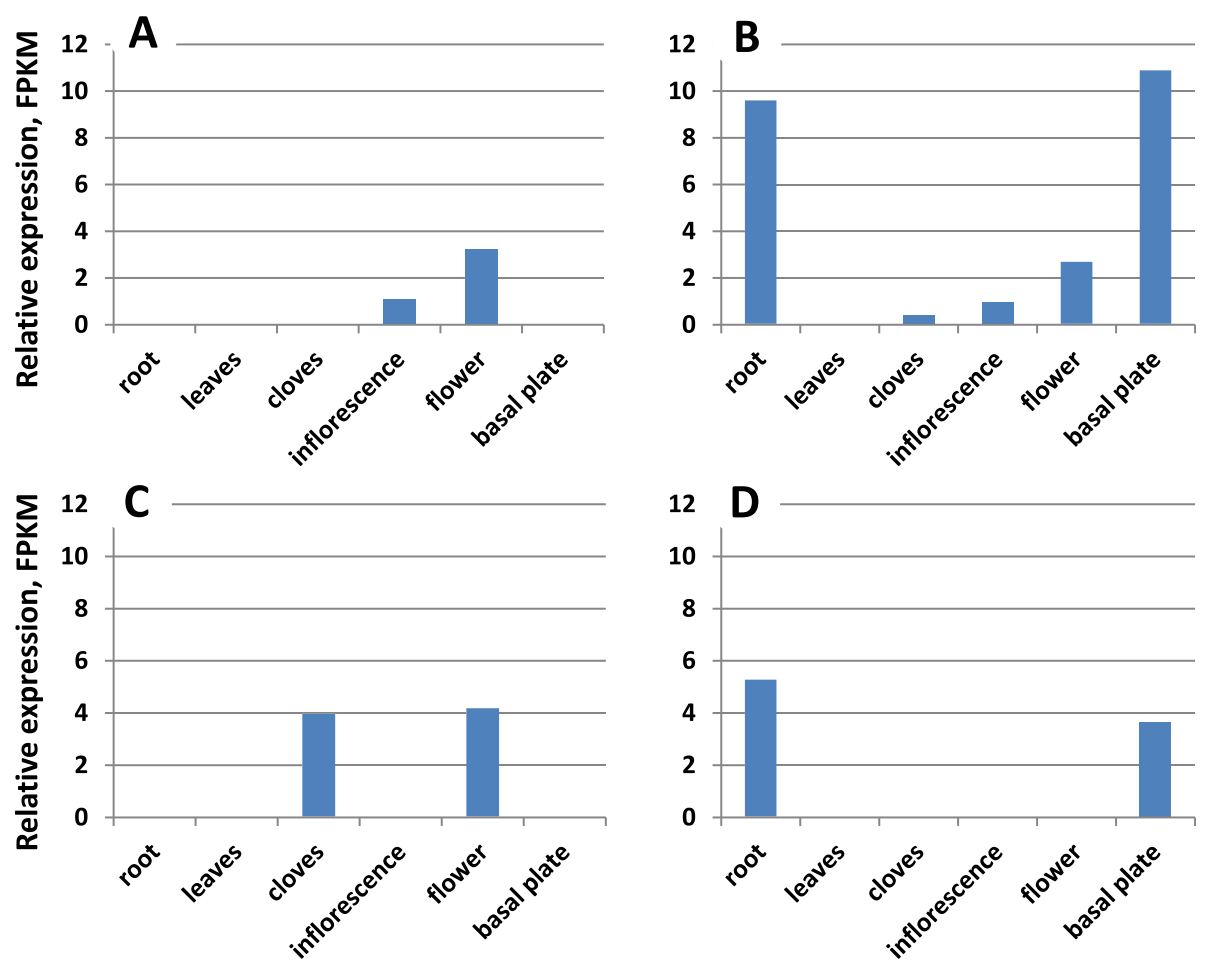

Figure 4 Differential expression of four FT orthologues in vegetative and reproductive organs of fertile garlic: (A) comp149377_c0_seq1, (B) comp32165_c0_seq1, (C) comp82533_c0_seq1, (D) comp4812_c0_seq1.

enzymes involved in sulfur metabolism in different organs of the garlic plant (Table 4). Isoforms of OASS varied significantly in their distribution in the garlic organs (Figure 5). Over-representation was notable in the cloves, roots, basal plate and leaves, whereas in the reproductive tissues, most of the annotated isoforms were less abundant. The isoforms were clustered into six groups, with clear expression patterns in the different organs. For instance, within cluster \#3 (highlighted in light blue in Figure 5), the three variants were enriched exclusively in cloves. The 10 variants and isoforms of SAT were also differentially expressed in the six garlic

Table 4 Variants of nine enzymes involved in sulfur metabolism found in the garlic transcriptome

\begin{tabular}{ll}
\hline Enzyme name & Number of variants \\
\hline Ferredoxin-sulfite reductase & 2 \\
ATP sulfurylase & 2 \\
Cysteine synthase & 27 \\
Serine acetyltransferase & 10 \\
Glutamate-cysteine ligase & 5 \\
Glutathione synthetase & 4 \\
Adenosine 5'-phosphosulfate reductase & 8 \\
O-acetylserine(thiol)-lyase & 26 \\
Gamma-glutamyl transferase & 22 \\
\hline
\end{tabular}

organs (data not shown), but organ clustering differed from that of OASS.

Numerous genes have been reported in the organosulfur biosynthesis pathways in garlic and onion. These include ATP sulfurylase, sulfite reductase, CS, SAT, glutamate cysteine ligase and glutathione synthase from garlic [27], and ferredoxin-sulfite reductase ( $\mathrm{SiR}$ ), adenosine 5'-phosphosulfate reductase (APSR), OASS, glutamylcysteine synthase, glutathione synthase, and $\gamma$-glutamyl transpeptidase from the onion bulb [52,53]. Our transcriptome analysis suggests that in addition to a large repertoire of enzyme isoforms, the pathways of sulfur metabolism and translocation of organosulfur compounds between garlic organs are diverse: the cysteine sulfoxides are synthesized primarily in the leaves and roots, and are then translocated to the developing cloves. Further and more intensive studies combining biochemistry and genetic regulation of these pathways may improve our understanding of alliin production and translocation in garlic and probably other Allium plants.

\section{GO classification of garlic viruses}

Garlic crops suffer heavy losses due to viral infections and other pests, and elimination of these viruses is particularly difficult. Moreover, due to the standard vegetative propagation of this crop, viruses are transmitted 


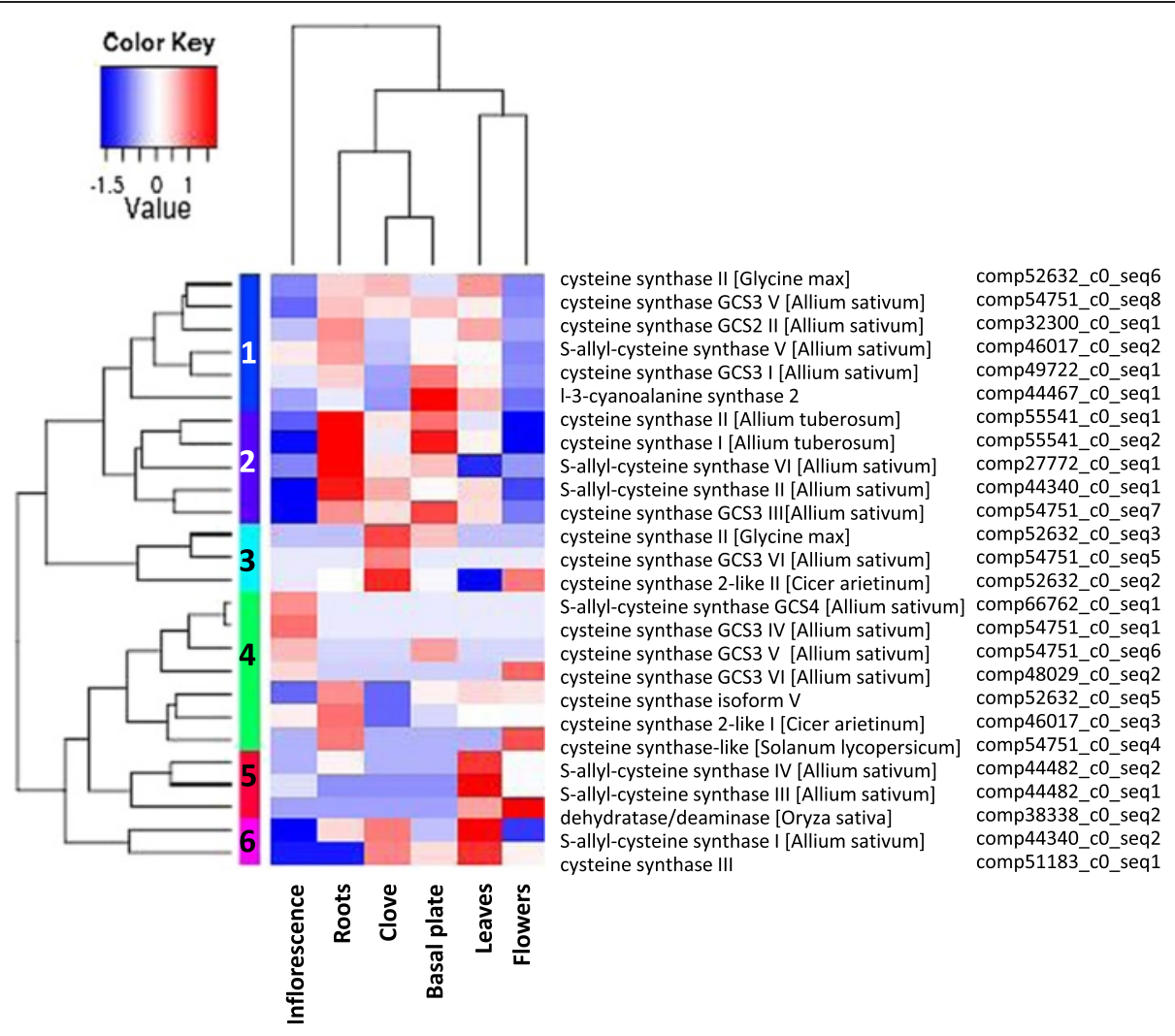

Figure 5 Hierarchical cluster analysis of gene-expression patterns of 26 annotated variants and isoforms of O-acetylserine(thiol)-lyase (OASS) in six vegetative and reproductive organs of garlic. The heat map shows the relative expression levels of each variant (rows) in each organ (columns). Expression values (FPKM) are log2-transformed and then median-centered by variant.

from one generation to the next [54]. The present transcriptome analysis was not designed to specifically search for garlic viruses. However, gene annotation revealed the presence of at least four known garlic allexiviruses: $\mathrm{A}, \mathrm{C}, \mathrm{E}$ and $\mathrm{X}$. Interestingly, we were unable to find transcripts similar to the most common and agriculturally significant potyviruses leek yellow stripe virus (LYSV) and onion yellow dwarf virus (OYDV) (Table 5). In onion, iris yellow spot tospovirus (IYSV) is not

Table 5 List of known viruses annotated in garlic transcriptome

\begin{tabular}{ll}
\hline Virus & Total read count \\
\hline Garlic virus C & 30,239 \\
Garlic virus A & 109,591 \\
Garlic virus E & 21,460 \\
Garlic virus X & 19,161 \\
Garlic latent virus & 0 \\
Leek yellow stripe virus & 0 \\
Onion yellow dwarf virus & 0 \\
Turnip mosaic virus & 0 \\
\hline
\end{tabular}

Relatively high expression of the allexiviruses $A, C, E$ and $X$ was found, while no sequence matches to garlic potyviruses were annotated. transmitted by seeds [55], but it is not known whether potyviruses of garlic are. Our experimental genotype \#87 was propagated and grown from a single seed in an insect-proof screenhouse, isolated from garlic and onion fields. Hence it might have been free of these viruses due to absence of inoculation. On the other hand, rather high amounts of the allexiviruses were identified in all tested organs, probably due to transmission via seeds. The highest amounts of viral RNA were identified in the roots and cloves. In contrast, flowers expressed only $1-$ $10 \%$ of the total viral RNA amount, and the lowest number of reads (1-4\%) was found in the leaves (Figure 6). This new information on the distribution of allexiviruses among the various plant organs provides a solid basis for future research and development of effective detection methods. In practice, leaves are often sampled for viral infection. It is evident that, at least for garlic allexiviruses, such sampling might result in erroneous estimations of viral infection, due to the relatively low titer in this organ.

Our initial results suggest that transcriptome tools provide more sensitive detection methods for new and known viruses in garlic, and shed new light on plant-pathogen interactions and virus distribution in plant tissues. 


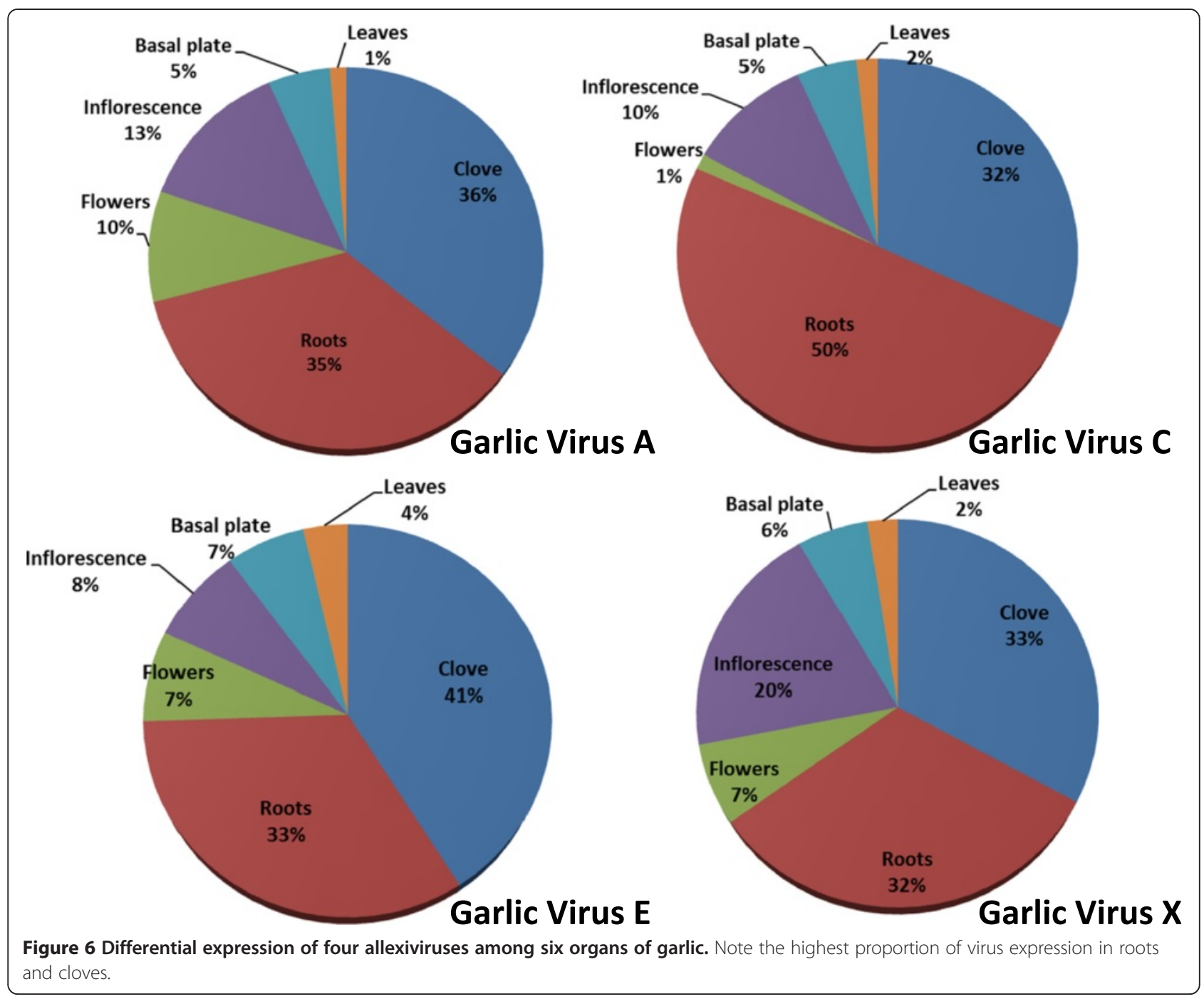

\section{Conclusions}

De-novo assembly of the transcriptome for fertile garlic provides a powerful tool for studying physiological, biochemical and genetic processes in garlic and other $\mathrm{Al}$ lium crops. The first organ-specific transcript catalogue was generated herein using relatively long reads (MiSeq 250), based on six labeled libraries from vegetative and reproductive tissues. We created two catalogues: (1) an extensive transcriptome consisting of 240,000 sequences, and (2) an abundant transcriptome of 102,000 contigs, which were annotated and analyzed for GO and metabolic pathways. These datasets provide versatile resources for garlic genome research, and can be used to associate the transcriptome to developmental processes, understand the regulatory network of these processes, trace the expression profiles of individual genes, and identify reference genes for quantitative expression analyses in various organs and tissues. This tool is especially important in the elucidation of mechanisms of floral induction, micro- and megasporogenesis, male sterility and seed production in garlic. It will also be useful in the development of new methods for virus detection, as well as in the study of plant-pathogen interactions.

In the future, garlic "omics" studies will facilitate the development of user-friendly, efficient, transferable, and codominant markers such as SNPs, to be applied in current and future breeding programs. Molecular assisted breeding applications will be used in the selection of genotypes with desired traits, produced from true seeds.

\section{Methods}

Plant material

Fertile garlic clone \#87 was derived from a single seed produced in 2004; further vegetative propagation guaranteed clonal uniformity. The plants were grown and propagated at the ARO, The Volcani Center, Bet Dagan, Israel. Freshly harvested bulbs were cured and stored under ambient conditions from July to September 2012 
in an open shed. Following visual inspection, healthy looking propagules were transferred and stored under controlled conditions at $4^{\circ} \mathrm{C}$ and a $\mathrm{RH}$ of $65-70 \%$ for 8 weeks. In November, healthy cloves were disinfected and planted in a net-house in a medium consisting of 50:25:25 (v/v/v) volcanic tuff particles, compost, and ground coconut peel. The plants were fertigated regularly using "Shefer" liquid fertilizer (N:P:K = 59:35:94 g L- ${ }^{1}$, Dshanim, Israel).

The experimental design is presented in Figure 7. Tissue samples were collected in three biological replicates between March and July 2013 from (1) the bulb basal plate; (2) green foliage leaves and scape; (3) roots; (4) young inflorescences with developing flower primordia, spathe removed; (5) individual flowers at various stages of development, and (6) cloves (Figure 8). The freshly sampled tissues were immediately dipped in liquid nitrogen and stored at $-80^{\circ} \mathrm{C}$ until RNA extraction.

\section{RNA isolation and sequencing procedures}

Total RNA was isolated from flowers and inflorescences using RNeasy Mini Kit (Qiagen, Hilden, Germany) according to the manufacturer's instructions. For the vegetative tissues, the CTAB protocol was used [56]. RNA extract quality was verified using the Agilent 2100 Bioanalyzer with a minimum RNA integrated number value of 7 .

Total RNA samples were shipped to the Roy J. Carver Biotechnology Center, W.M. Keck Center for Comparative and Functional Genomics, Urbana, IL, USA for library preparation and sequencing. The RNAseq libraries were prepared with Illumina's TruSeq RNA Sample Prep Kit (www.Illumina.com) with one modification: RNA was randomly primed but not chemically fragmented. The pool was quantified by qPCR and sequenced for 251 cycles from each end of the fragments on a MiSeq v3 platform using a MiSeq sequencing kit, version 3, with a relatively long read length of $250 \mathrm{nt}$, and analyzed with Casava 1.8 (pipeline 1.8).

\section{De novo sequence assembly}

Raw reads were subjected to a cleaning procedure using the FASTX Toolkit (http://hannonlab.cshl.edu/fastx_toolkit/index.html, version 0.0.13.2) including: (1) trimming read-end nucleotides with quality scores $<30$ using fastq_quality_trimmer; (2) removing read pairs if either one

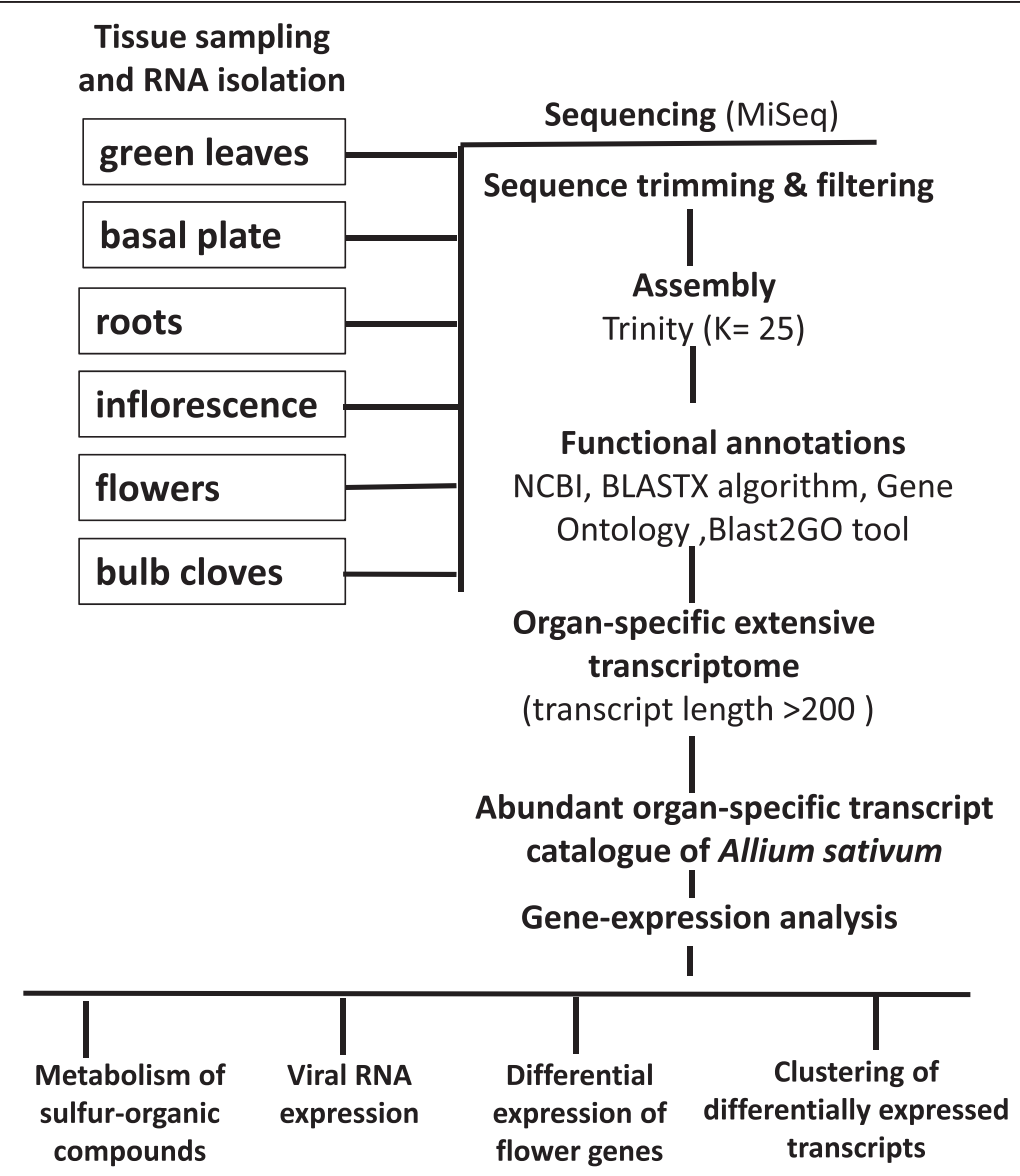

Figure 7 Experimental design of the sequencing, assembly, annotation, construction and analyses of the organ-specific transcriptome catalogues of Allium sativum. 


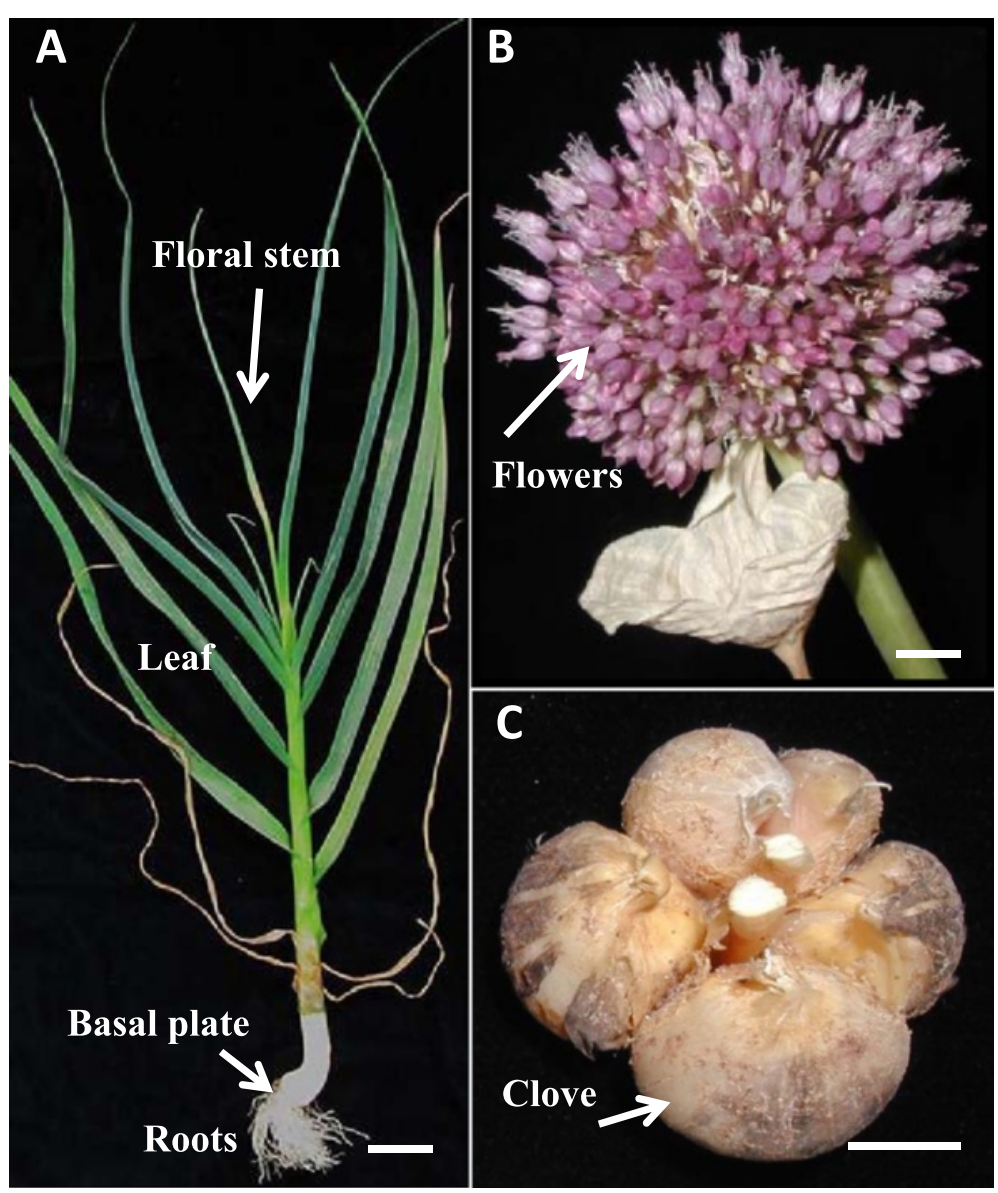

Figure 8 Organography of tissue sampling in fertile garlic genotype \#87. Samples were collected in March-July 2013. (A) Image of the entire plant at vegetation stage, prior to flowering; $b a r=7 \mathrm{~cm}$. (B) Fully developed multi-flower inflorescence; $b a r=0.8 \mathrm{~cm}$. (C) Mature bulb consisting of a few cloves; $b a r=1 \mathrm{~cm}$.

had less than $70 \%$ base pairs with quality score $\leq 30$ using fastq_quality_filter. A total of 28.4 million cleaned pairedend reads, obtained after processing and cleaning, were assembled de novo using Trinity software (version trinityrnaseq_r2013_08_14 [57] with default parameters and 25 mer k-mer size. For assembly, the initial parameters of Trinity were set as follows:-seqType fq -JM 400G -CPU 10 -bflyHeapSpaceMax 400G -bflyHeapSpaceInit 400G -bflyCPU 10. The assembled sequences that shared a number of k-mers (the set of isoforms of a gene) were referred to as "contigs". The sets of all sequences that shared at least one k-mer were referred to as components.

The resulting de novo assembly was used to generate two transcriptomes: the "extensive transcriptome" and the "abundant transcriptome". The extensive transcriptome consisted of all of the contigs, whereas the abundant transcriptome included only the contigs with count per sample $\geq 10$. CD-HIT (http://www.bioinformatics. $\mathrm{org} / \mathrm{cd}$-hit/) with $95 \%$ global sequence identity was used to cluster the sequences of both transcriptomes.
For the abundant transcriptome compilation, filtration of the likely contig artifacts and low expressed contigs was carried out as follows: (1) abundance estimates were calculated for each contig using the RSEM software package [58]; (2) only contigs representing more than $1 \%$ of the per-component (IsoPct) expression level were retained; (3) finally, contigs with total mapped reads of less than 10 were removed.

\section{Data availability}

The sequencing data were deposited in the NCBI Sequence Read Archive (SRA) database as bioproject PRJNA243415 [GenBank:SRR1219535, GenBank:SRR 1219644, GenBank:SRR1219646, GenBank:SRR1219796, GenBank:SRR1219989, GenBank:SRR1220207].

\section{Sequence similarity and functional annotation}

To assess the similarity of the garlic transcriptome to those of other model and closely related species, analysis of sequence similarity was performed using the BLAST 
(Basic Local Alignment Search Tool) algorithm with an E-value cut-off of $10^{-5}$ [59].

Comparison to the transcriptomes of the garlic renewal bud [27], onion [26] and Tulipa and Lilium [24,25] was performed using the BLASTN algorithm, which allows comparison using a nucleotide query. In addition, the BLASTX algorithm was used to search protein databases using a translated nucleotide query for the comparison of the assembled contigs with sequences deposited in the databases of Arabidopsis (http://www.arabidopsis.org), Oryza sativa (www.phytozome.org) and NCBI nonredundant (nr) proteins (http://www.ncbi.nlm.nih.gov; http://www.ncbi.nlm.nih.gov/genbank/statistics). Blosum62 matrix was used for BLASTX (when searching against a protein database with a DNA query), TBLASTN (when searching for translated nucleotide databases with a protein query) and TBLASTX (when searching for translated nucleotide databases with a translated nucleotide query). BLASTN was executed using the default parameters of the Blastall program (version 2.2.25).

GO annotations were assigned using Blast2GO [60] based on an nrNCBI search. GO provides a structured and controlled terminology to describe the gene products, according to three categories: molecular function (refers to the biochemical activity of a gene product without stating the location of the event), biological process (refers to the biological objective to which the gene product contributes), and cell component (refers to the place in the cell where a gene product is active). GO-enrichment analysis was performed using Fisher's Exact Test of Blast2GO software. Analysis was carried out by comparing the GO terms in the test sample to the GO terms in a background reference. A false discovery rate [61] with corrected P-value of less than 0.05 was set as the threshold. The REViGO web server was used for visualization of the GO terms in a semantic similarity-based scatterplot (http://revigo.irb.hr/ [62]).

In the search for orthologous genes involved in flowering induction or sulfur metabolism, 22 candidate sequences were retrieved from databases in the NCBI (Additional file 4: Table S2). Sequences were compared with the abundant garlic transcriptome using TBLASTN, with an E-value cut-off of $10^{-5}$.

\section{Abundance estimation and differential expression analysis}

The cleaned reads from each organ were aligned separately with the abundant transcriptome assembly using the Bowtie aligner [63]. Following the Trinity protocol [64], the abundance estimation was calculated using the run_RSEM_align_n_estimate.pl script Perl. Since reads are generally shorter than transcripts from which they are derived, a single read may map to multiple genes and isoforms, thus complicating expression analyses.
Therefore, the transcript quantification from RNA-Seq data was performed using the Expectation-Maximization method (RSEM), which handles read mapping uncertainty with a statistical model by estimating maximum likelihood expression levels $[58,65]$. Bioconductor EdgeR package [66] in the $R$ environment was used to identify differentially expressed transcripts for each pair of samples (15 comparisons overall), based on the count estimations for each contig. Transcripts that were more than fourfold differentially expressed with false discovery- corrected statistical significance of at most 0.001 were considered differentially expressed.

\section{Cluster analysis}

The expression patterns of the transcripts in different organs were studied using cluster analysis of the differentially expressed transcripts in at least one pairwise sample comparison. Following the Trinity protocol [64], expression normalization was calculated using TMM (trimmed mean of M-values), following FPKM (fragments per feature kilobase per million reads mapped) calculations. Then, hierarchical clustering of transcripts and samples was performed and clusters were extracted using R scripts.

\section{Analysis of viral genome sequences}

Eight genome sequences of garlic viruses were downloaded from the NCBI genome database (ftp://ftp.ncbi. nlm.nih.gov/genomes/Viruses/), including: leek yellow stripe virus (LYSV) (NC_004011.1), onion yellow dwarf virus (OYDV) (NC_005029.1), garlic virus $C$ ( $\mathrm{NC}_{-}$ 003376.1), garlic virus $A$ (NC_003375.1), garlic virus $\bar{E}$ (NC_004012.1), garlic virus $X$ (NC_001800.1), garlic latent virus (NC_003557.1) and turnip mosaic virus (TMV) (NC_002509.2). The cleaned reads, obtained separately from each organ, were aligned independently to the eight viruses using Bowtie2 aligner (version 2.1.0; [67]) and the counts were estimated using the SAM tools software [68].

\section{Additional files}

Additional file 1: Figure S1. Representation of enriched biological processes and functions in clusters \#1 and 2 (Figure 3), as analyzed by Blast2GO and REViGO tools. The biological process terms are arranged in semantic space and colored by semantic positioning on the X-axis, the size of bullet points by significance (log10 of P-value). Only GO terms with contig counts higher than $1 \%$ are shown. (a) Enriched biological processes and molecular functions in cluster \#1, predominantly in the basal plate and roots. The main processes include responses to various types of stress and metabolite transport. (b) Enriched biological processes and molecular functions in cluster \#2, predominantly in the basal plate. The main processes include catalytic activity and interaction with virus.

Additional file 2: Figure S2. Representation of enriched biological processes and functions in clusters \#3 and 4 (Figure 3), as analyzed by Blast2GO and REViGO tools. The biological process terms are arranged in 
semantic space and colored by semantic positioning on the $X$-axis, size of bullet points by significance ( $\log 10$ of P-value). Only GO terms with contig counts higher than 1\% are shown. (a) Enriched biological processes and molecular functions in cluster \#3, mainly in the reproductive organs. The two main groups include the pathways of florogenesis/gametogenesis and DNA replication/modification. (b) Enriched biological processes and molecular functions in cluster \#4, mainly in the foliage leaves. The main processes include response to light quality and intensity, photosynthesis, and biosynthesis of the secondary metabolites.

Additional file 3: Table S1. List of the 12 most abundant proteins in the vegetative and reproductive organs of garlic. The expression level (FPKM) was calculated via the expectation maximization method and TMM normalization (trimmed mean of M-value normalization method) was applied (see Materials and Methods). The annotation was based on the comparison with nrNCBI using BLASTX. Note over-representation of three non-annotated proteins in leaves and flowers.

Additional file 4: Table S2. Candidate sequences of genes involved in flowering and sulfur metabolism, retrieved from NCBI databases (April 2014).

\section{Competing interests}

This study was supported by the company Classeed Ltd., which provided funding for the experimental work, data analysis, publication fees and salaries for ESM, TBM, SK and CG. This publication is fully authorized by the company.

\section{Authors' contributions}

RK and AS designed the experiment and drafted the manuscript. AF carried out the bioinformatics analysis. ESM, TBM, SRS, IE, SK and CG conducted the experimental work. HDR and DE conceived the study, and contributed to the writing of the manuscript. This research is a part of the graduate studies of ESM and TBM. All authors have read and approved the final manuscript.

\section{Acknowledgements}

We wish to thank Dr. Paul Arens (Wageningen University, The Netherlands) for scientific revision and suggestions during the preparation of the manuscript.

\section{Author details}

'Institute of Plant Sciences, ARO, The Volcani Center, Bet Dagan, Israel. ${ }^{2}$ Robert H. Smith Faculty of Agricultural, Food, and Environmental Quality Sciences, The Hebrew University of Jerusalem, Jerusalem, Israel. ${ }^{3}$ Classeed Ltd., Gibraltar, British. ${ }^{4}$ Institute of Postharvest and The Food Sciences, ARO, The Volcani Center, Bet Dagan, Israel.

\section{Received: 24 June 2014 Accepted: 31 December 2014}

Published online: 22 January 2015

\section{References}

1. Augusti KT. Therapeutic and medicinal values of onions and garlic. In: Brewster JL, Rabinowitch HD, editors. Onions and Allied Crops. Volume III. Biochemistry, Food Science, and Minor Crops. Boca Raton, FL: CRC Press, Inc; 1990. p. 93-108.

2. Kamenetsky R. Garlic: botany and horticulture. In: Janick J, editor. Horticultural Reviews. Volume 33. New Jersey: Wiley; 2007. p. 123-72.

3. Block E. Garlic and Other Alliums: The Lore and The Science. Cambridge, UK: Royal Society of Chemistry; 2010.

4. Etoh T, Simon PW. Diversity, fertility and seed production of garlic. In: Rabinowitch HD, Currah L, editors. Allium Crop Sciences: Recent Advances. Wallingford, UK: CABI; 2002. p. 101-17.

5. Pooler MR, Simon PW. Garlic flowering in response to clone, photoperiod, growth temperatures and cold storage. HortSci. 1993;28:1085-6.

6. Pooler MR, Simon PW. True seed production in garlic. Sex Plant Reprod. 1994;7:282-6.

7. Jenderek MM, Hannan RM. Variation in reproductive characteristics and seed production in the USDA garlic germplasm collection. HortSci. 2004;39:485-8.

8. Kamenetsky R, London Shafir I, Khassanov F, Kik C, van Heusden AW, Vrielink-van Ginkel $\mathrm{M}$, et al. Diversity in fertility potential and organo-sulphur compounds among garlics from Central Asia. Biodivers Conserv. 2005;14:281-95.

9. Kamenetsky R, London Shafir I, Zemah H, Barzilay A, Rabinowitch HD. Environmental control of garlic growth and florogenesis. J Am Soc Hortic Sci. 2004;129:144-51.
10. Zewdie Y, Havey MJ, Prince JP, Jenderek MM. The first genetic linkages among expressed regions of the garlic genome. J Am Soc Hortic Sci. 2005;130:569-74.

11. Shemesh E, Scholten O, Rabinowitch HD, Kamenetsky R. Unlocking variability: inherent variation and developmental traits of garlic plants originated from sexual reproduction. Planta. 2008;227:1013-24.

12. Shemesh Mayer E, Winiarczyk K, Błaszczyk L, Kosmala A, Rabinowitch HD, Kamenetsky R. Male gametogenesis and sterility in garlic (Allium sativum L): barriers on the way to fertilization and seed production. Planta. 2013;237:103-20.

13. Rotem N, Shemesh E, Peretz Y, Akad F, Edelbaum O, Rabinowitch HD, et al. Reproductive development and phenotypic differences in garlic are associated with expression and splicing of LEAFY homologue gaLFY. J Exp Bot. 2007;58:1133-41.

14. Rotem N, David-Schwartz R, Peretz Y, Sela I, Rabinowitch HD, Flaishman M, et al. Flower development in garlic: the ups and downs of gaLFY expression. Planta. 2011;233:1063-72.

15. Randle WM, Lancaster JE. Sulphur compounds in Alliums in relation to flavor quality. In: Rabinowitch HD, Currah L, editors. Allium Crop Science, Recent Advances. Wallingford Oxon, UK: CABI; 2002. p. 329-56.

16. Jones MG, Hughes J, Tregova A, Milne J, Tomsett AB, Collin HA. Biosynthesis of the flavour precursors of onion and garlic. J Exp Bot. 2004;55:1903-18.

17. Jones MG, Collin HA, Tregova A, Trueman L, Brown L, Cosstick R. The biochemical and physiological genesis of alliin in garlic. Med Aromatic Plant Sci Biotechnol. 2007;1:21-4.

18. Ricroch A, Yockteng R, Brown S, Nadot S. Evolution of genome size across some cultivated Allium species. Genome. 2005;48:511-20.

19. Arumuganathan K, Earle ED. Nuclear DNA content of some important plant species. Plant Mol Biol Report. 1991;9:208-18.

20. Gai S, Zhang Y, Mu P, Liu C, Liu S, Dong L, et al. Transcriptome analysis of tree peony during chilling requirement fulfillment: assembling, annotation and markers discovering. Gene. 2012;497:256-62.

21. Ophir R, Sherman A, Rubinstein M, Eshed R, Sharabi Schwager M, Harel-Beja R, et al. Single-nucleotide polymorphism markers from de-novo assembly of the pomegranate transcriptome reveal germplasm genetic diversity. PLOS ONE. 2014;9:e88998.

22. Su CL, Chao YT, Alex Chang YC, Chen WC, Chen CY, Lee AY, et al. De novo assembly of expressed transcripts and global analysis of the Phalaenopsis Aphrodite transcriptome. Plant Cell Physiol. 2011;52:1501-14.

23. Sablok G, Fu Y, Bobbio V, Laura M, Rotino GL, Bagnaresi P, et al. Fuelling genetic and metabolic exploration of $\mathrm{C} 3$ bioenergy crops through the first reference transcriptome of Arundo donax L. Plant Biotechnol J. 2014;12:554-67.

24. Shahin A, Van Kaauwen M, Esselink D, Bargsten JW, Van Tuyl JM, Visser RGF, et al. Generation and analysis of expressed sequence tags in the extreme large genomes Lilium and Tulipa. BMC Genomics. 2012;13:640.

25. Shahin A, Van Gurp T, Peters SA, Visser RGF, Van Tuyl JM, Arens P. SNP markers retrieval for a non-model species: a practical approach. BMC Res Notes. 2012;1:79.

26. Duangjit J, Bohanec B, Chan AP, Town CD, Havey MJ. Transcriptome sequencing to produce SNP-based genetic maps of onion. Theor Appl Genet. 2013;126:2093-101.

27. Sun X, Zhou S, Meng F, Liu S. De novo assembly and characterization of the garlic (Allium sativum) bud transcriptome by Illumina sequencing. Plant Cell Rep. 2012;31:1823-8.

28. Ipek M, Ipek A, Aydogan C, Senalik D, Simon PW. Differentially expressed genes in leaf, meristematic and flower tissues of garlic. Acta Horticult. 2012;969:53-7.

29. Cho H, Davis J, Li X, Smith KS, Battle A, Montgomery SB: High-resolution transcriptome analysis with long-read RNA sequencing. [http://arxiv.org/abs/ 1405.7316]

30. Magallón S, Castillo A. Angiosperm diversification through time. Am J Bot. 2009:96:349-65.

31. Meerow A. Taxonomy and phylogeny. In: Kamenetsky R, Okubo H, editors. Ornamental Geophytes: from Basic Science to Sustainable Production. Boca Raton, FL: CRC Taylor and Francis Group; 2012. p. 17-56.

32. Chase MW, Fay MF, Devey DSO, Maurin N, Rønsted TJ, Davies Y, et al. Multigene analyses of monocot relationships: a summary. In: Columbus JT, Friar EA, Hamilton CW, Porter JM, Prince LM, Simpson MG, editors. Monocots: Comparative Biology and Evolution. Volume 1, excluding Poales. Claremont, CA: Rancho Santa Ana Botanic Garden; 2006. p. 63-75.

33. Aceituno FF, Moseyko N, Seung YR, Rodrigo AG. The rules of gene expression in plants: organ identity and gene body methylation are key factors for regulation of gene expression in Arabidopsis thaliana. BMC Genomics. 2008;9:438. 
34. Libault M, Farmer A, Joshi T, Takahashi K, Langley RJ, Franklin LD, et al. An integrated transcriptome atlas of the crop model Glycine max, and its use in comparative analyses in plants. Plant J. 2010;63:86-99.

35. Lei W, Xie W, Chen Y, Tang W, Yang J, Ye R, et al. A dynamic gene expression atlas covering the entire life cycle of rice. Plant J. 2010;61:752-66.

36. Wei LQ, Xu WY, Deng ZY, Su Z, Xue Y, Wang T. Genome-scale analysis and comparison of gene expression profiles in developing and germinated pollen in Oryza sativa. BMC Genomics. 2010;11:338.

37. Fujita M, Horiuchi Y, Ueda Y, Mizuta Y, Kubo T, Yano K, et al. Rice expression atlas in reproductive development. Plant Cell Physiol. 2010;51:2060-81.

38. Kamenetsky R. Biodiversity of geophytes: phytogeography, morphology and survival strategies. In: Kamenetsky R, Okubo H, editors. Ornamental Geophytes: from Basic Science to Sustainable Production. Boca Raton, FL: CRC Press, Taylor and Francis Group; 2012. p. 57-76.

39. Rabinowitch HD, Fridovich I. Superoxide radicals, superoxide dismutases and oxygen toxicity in plants. Photochem Photobiol. 1983;37:679-90.

40. Brewster JL. Onions and Other Alliums. 2nd ed. Wallingford, UK: CABl; 2008.

41. Okubo H. Dormancy. In: Kamenetsky R, Okubo H, editors. Ornamental Geophytes: from Basic Science to Sustainable Production. Boca Raton, FL: CRC Taylor and Francis Group; 2012. p. 233-60.

42. Tremblay R, Colasanti J. Floral induction. In: Ainsworth C, editor. Annual Plant Reviews: Flowering and Its Manipulation. Volume 20. Oxford: Blackwell Publishing; 2006. p. 28-48.

43. Tsuji H, Taoka Kl, Shimamoto K. Regulation of flowering in rice: two florigen genes, a complex gene network, and natural variation. Curr Opin Plant Biol. 2011;14:45-52

44. Simpson GG, Dean C. Arabidopsis, the Rosetta stone of flowering time? Science. 2002;296:285-9.

45. Song $\mathrm{YH}$, Ito S, Imaizumi T. Similarities in the circadian clock and photoperiodism in plants. Curr Opin Plant Biol. 2010;13:594-603.

46. Taylor A, Massiah AJ, Thomas B. Conservation of Arabidopsis thaliana photoperiodic flowering time genes in onion (Allium cepa L.). Plant Cell Physiol. 2010;51:1638-47.

47. Lee R, Baldwin S, Kenel F, McCallum J, Macknight R. FLOWERING LOCUS T genes control onion bulb formation and flowering. Nat Commun. 2013;4:1-9.

48. Shalit A, Rozman A, Goldshmidt A, Alvarez JP, Bowman JL, Eshed E, et al. The flowering hormone florigen functions as a general systemic regulator of growth and termination. Proc Natl Acad Sci U S A. 2009;106:8392-7.

49. Noy-Porat T, Cohen D, Mathew D, Eshel A, Kamenetsky R, Flaishman MA. Turned on by heat: differential expression of FT and LFY-like genes in Narcissus tazetta during floral transition. J Exp Bot. 2013;64:3273-84.

50. Abelenda JA, Navarro C, Prat S. From the model to the crop: genes controlling tuber formation in potato. Curr Opin Biotechnol. 2011;22:287-92.

51. Salsi E, Campanini B, Bettati S, Raboni S, Roderick SL, Cook PF, et al. A two-step process controls the formation of the bienzyme cysteine synthase complex. J Biol Chem. 2010;285:12813-22

52. Yaguchi S, Ono Y, Nakajima T, Yamauchi N, Shigyo M, Masamura N, et al. Biochemical and molecular analyses of amino acid and S-alk(en)yl-L-cystein sulfoxide production in Allium fistulosum-A. cepa monosomic addition lines. Acta Horticult. 2012;969:59-64.

53. McManus MT, Joshi S, Searle B, Pither-Joyce M, Shaw M, Leung S, et al. Genotypic variation in sulfur assimilation and metabolism of onion (Allium cepa L.). III. Characterization of sulfite reductase. Phytochemistry. 2012;83:34-42.

54. Salomon R. Virus diseases in garlic and the propagation of virus-free plants. In: Rabinowitch HD, Currah L, editors. Allium Crop Science: Recent Advances. Wallingford Oxon, UK: CABl; 2002. p. 311-27.

55. Kritzman A, Raccah B, Gera A: Transmission of Iris Yellow Spot Tospovirus. In Proceedings of the 7th International Symposium "Thrips, Plants, Tospoviruses: the Millennial Review", 2-7 July 2001; Calabria, Italy. Edited by Marullo R, Mound L. Canberra, Australia: CSIRO; 2002:95-98.

56. Chang S, Puryear J, Cairney J. A simple and efficient method for isolating RNA from pine trees. Plant Mol Biol Report. 1993;11:113-6.

57. Grabherr MG, Haas BJ, Yassour M, Levin JZ, Thompson DA, Amit I, et al. Full-length transcriptome assembly from RNA-Seq data without a reference genome. Nat Biotechnol. 2011;29:644-52.

58. Li B, Dewey CN. RSEM: accurate transcript quantification from RNA-Seq data with or without a reference genome. BMC Bioinformatics. 2011;12:323.

59. Altschul SF, Gish W, Miller W, Myers EW, Lipman DJ. Basic local alignment search tool. J Mol Biol. 1990;215:403-10.
60. Conesa AS, Götz JM, Garcia-Gomez J, Talon TM, Robles M. Blast2GO: a universal tool for annotation, visualization and analysis in functional genomics research. Bioinformatics. 2005;21:3674-6.

61. Benjamini Y, Hochberg Y. Controlling the false discovery rate: a practical and powerful approach to multiple testing. J R Stat Soc Ser B. 1995;57:289-300.

62. Supek F, Bosnjak M, Skunca N, Smuc T. REVIGO summarizes and visualizes long lists of gene ontology terms. PLoS ONE. 2011;6:e21800.

63. Langmead B, Trapnell C, Pop M, Salzberg SL. Ultrafast and memory-efficient alignment of short DNA sequences to the human genome. Genome Biol. 2009;10:R25.

64. Haas BJ, Papanicolaou A, Yassour M, Grabherr M, Blood PD, Bowden J, et al. De novo transcript sequence reconstruction from RNA-seq using the Trinity platform for reference generation and analysis. Nat Protoc. 2013;8:1494-512.

65. Li B, Ruotti V, Stewart RM, Thomson JA, Dewey CN. RNA-Seq gene expression estimation with read mapping uncertainty. Bioinformatics. 2010;26:493-500.

66. Robinson MD, McCarthy DJ, Smyth GK. edgeR: a Bioconductor package for differential expression analysis of digital gene expression data. Bioinformatics. 2010;26:139-40.

67. Langmead B, Salzberg S. Fast gapped-read alignment with Bowtie 2 . Nat Methods. 2012;9:357-9.

68. Li H, Handsaker B, Wysoker A, Fennell T, Ruan J, Homer N, et al. The Sequence alignment/map (SAM) format and SAMtools. Bioinformatics. 2009;25:2078-9.

\section{Submit your next manuscript to BioMed Central and take full advantage of:}

- Convenient online submission

- Thorough peer review

- No space constraints or color figure charges

- Immediate publication on acceptance

- Inclusion in PubMed, CAS, Scopus and Google Scholar

- Research which is freely available for redistribution 